\title{
APLICACIÓN DE LA METODOLOGÍA QFD EN EL DESARROLLO DE UNA IMPRESORA 3D
}

\section{APPLICATION OF THE QFD METHODOLOGY IN THE DEVELOPMENT OF A 3D PRINTER}

\author{
Ing. Efraín Andrés Rodríguez Gasca, Ing. Eliseo de Jesús Cortés Torres \\ PhD. César Augusto Peña Cortés
}

Universidad de Pamplona, Programa de Ingeniería Mecatrónica Ciudadela Universitaria. Pamplona, Norte de Santander, Colombia. Tel.: (+57 7) 568 5303, Fax: (+57 7) 568 5303, Ext. 144.

E-mail: \{efrain.rodriguez, eliseo.cortes, cesarapc\}@unipamplona.edu.co

\begin{abstract}
Resumen: Este artículo presenta el desarrollo de una impresora 3D bajo el enfoque de la metodología del desarrollo de la función de la calidad - QFD, se hace hincapié en las necesidades y requerimientos de los clientes, en este caso, los estudiantes de la facultad de ingenierías de la Universidad de Pamplona, teniendo como resultados el desarrollo de una ruta crítica en la planificación del proyecto, además de su diseño asistido por computadora - CAD, construcción y la puesta a punto del sistema.
\end{abstract}

Palabras clave: Desarrollo de la función de la Calidad, Planificación de proyectos, Diseño Mecatrónico, Robot paralelo, Impresión 3D.

\begin{abstract}
This paper carries out the development of a 3D printer under the focus of the QFD methodology, emphasis on the needs and requirements of customers, in this case, students of the faculty of engineering at the University of Pamplona, having as a result the development of a critical path in the project planning, in addition to its CAD design, construction and the system test.
\end{abstract}

Keywords: Quality Function Development, Planning, Project planning, Mechatronic Design, Parallel Robot, 3D Printer.

\section{INTRODUCIÓN}

Hoy en día no basta con generar productos y buscar un mercado para su venta, ahora se debe sumergir al consumidor dentro del proceso del proceso de diseño de los productos, tanto para satisfacer las necesidades para las cuales están concebidos, como para generar expectativas que sobrepasen lo pensado por los usuarios creando nuevas necesidades para que estos procesos sean versátiles e innovadores (Goetsch et al. 1993).

Una de las metodologías que permite tener en cuenta la opinión de los usuarios acerca de un producto antes de su concepción y que ha demostrado ser una herramienta eficaz es la metodología QFD (Desarrollo de la Función de la Calidad por sus siglas en ingles) (Kahraman et al. 2006).

En sistemas mecatrónicos donde la robótica juega un papel fundamental, existen múltiples factores que se deben tener en cuenta para llevar a buen término el desarrollo de los proyectos.

Existen factores como los costos, la estética, el control intuitivo o amigable que hacen de estas 
máquinas agradables al consumidor aumentando o incentivado su uso por personal sin conocimientos técnicos avanzados (Pertuz y Peña 2014), por otra parte se deben tener en cuenta otros aspectos como la funcionalidad, las prestaciones, ergonomía e incluso los beneficios sociales y la rentabilidad ofrecida por estos sistemas robotizados (Caballero A. et al., 2013).

Además del desarrollo de aplicaciones, máquinas, prototipos en el área de ingeniería, la metodología QFD se puede adaptar a proyectos de cualquier índole (Huang y Low, 2010), siendo una metodología flexible y adaptable a cualquier campo de investigación.

La necesidad en algunas regiones de contar con máquinas de prototipado para el desarrollo de modelos en 3D ha impartido una cultura de diseño y desarrollo de estas (Chancusi et al.; Bradley y Dorey; Kostakis et al., 2014).

Permitiendo mayor accesibilidad a las tecnologías de punta, mejor y mayor conocimiento de los fenómenos físicos, y a la creación de modelos didácticos para la impartición de clases (Rodríguez et al., 2012).

Las constantes mejoras en cuanto a velocidad, control cinemático, control dinámico, repetitividad, precisión y exactitud de los robots paralelos han incentivado a los investigadores al desarrollo de plataformas pluridisciplinares, también debido al constante desarrollo de necesidades basadas en la tecnología de prototipado $3 \mathrm{D}$, a su velocidad de producción, el estudio de nuevos materiales, han permitido brindar constantes avances en este campo de la industria (Osgouie 2008; Burisch, Soetebier, and Wrege 2004; Cervantes-Sánchez, Hernández-Rodríguez, and Rendón-Sánchez 2000; Cross 2014; Dallalibera and Ishiguro 2014; Do et al. 2012; Giberti, Cinquemani, and Ambrosetti 2013; Kim, Lee, and Yi 1997; Liu, Wang, and Pritschow 2006).

Es así como este trabajo recolecta toda la parte de metodología de investigación, para su posterior buena ejecución, el artículo está estructurado de la siguiente forma: en la sección 2 se encuentra la planificación del proyecto, en la sección 3 los criterios y requerimientos de la máquina basados en la metodología QFD, la sección 4 se encuentra el diseño estructural de la máquina, en la sección 5 se encuentran los resultados obtenidos y en la sección 6 las conclusiones.

\section{PLANIFICACIÓN Y EJECUCIÓN}

La planificación de un proyecto, tiene como objetivo hacer que todos los elementos que están inmersos dentro de él, se lleven a cabo correctamente (Torres et al., 2013).

Con la ayuda de un software, se desarrolló una ruta crítica a seguir para la concepción de la totalidad de la máquina, de esta manera se entiende que su diseño se basa en el modelo de estructura concurrente, en donde cada etapa del proyecto solo puede trabajar cuando la anterior ya está parcialmente culminada.

Teniendo estos criterios se empieza con la inclusión de datos en el software de la siguiente manera:

\subsection{Definición de tareas y/o recursos}

La definición de tareas se lleva a cabo teniendo en cuenta las etapas de desarrollo, en esta parte del trabajo se estructura, las actividades se deben implementar para que las tareas sean cumplidas a cabalidad, por ejemplo, para la documentación y estado del arte es imprescindible la búsqueda de artículos de investigación, además de búsquedas WEB y demás (Ver figura 1).

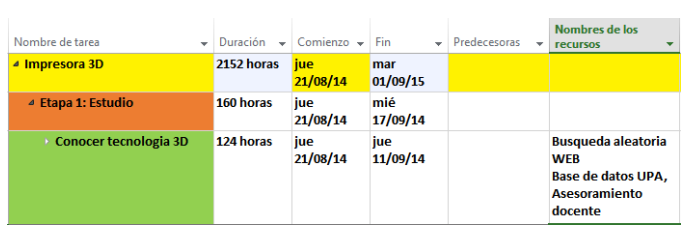

Fig. 1: Recursos necesarios para desarrollar las tareas (Ejemplo del uso de un programa de gestión de proyectos).

En otros casos, como el uso de talleres, máquinas y herramientas para la fabricación de piezas es fundamental, elaborar una lista de estos instrumentos, los cuales se deben tener en cuenta para el desarrollo de esta etapa del proyecto (ver figura 2).

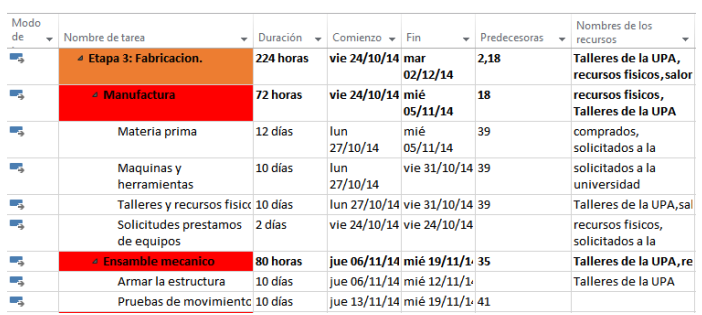

Fig. 2: Lista de recursos máquinas de acuerdo a la tarea que se debe realizar. 


\subsection{Duración de tareas y predecesoras}

La duración de tareas se estable en dependencia al tiempo que se requiera para su culminación, es así como se define un promedio de días de horas utilizadas para ellas, cuando ya se tiene su totalidad, se debe saber que el horario de trabajo para el proyecto está establecido en 8 horas diarias de trabajo, en un horario de 8 am a $12 \mathrm{~m}$ y de $2 \mathrm{pm}$ a 6 pm.

La herramienta llamada predecesora es utilizada para que las tareas que se consideran no realizables antes de otra no se puedan ejecutar, y por ende no se establezcan en la ruta crítica de la misma. En esta investigación de logro desarrollar un tipo de diseño de manera concurrente. Esta forma de planificar proyectos se llevó a cabo de manera satisfactoria, brindando así una ruta crítica de apoyo para soportar y establecer prioridades a la hora de ejecutar lo asignado.



Fig. 3: Diagrama de tiempo de la planificación del proyecto.

La ruta crítica, se presenta en la figura 3 como una escala de tiempo, y en la figura 4 como un diagrama de Gantt obtenidos mediante el software de planificación utilizado.



Fig. 4: Diagrama de Gantt de una tarea planificada.

\section{CRITERIOS Y CARACTERÍSTICAS DE LA MÁQUINA.}

Basados en la idea de introducir al cliente en el desarrollo de sus productos de consumo, se ha desarrollado una encuesta con población de 18 estudiantes de la Universidad de Pamplona, el proyecto obtuvo un grado de aceptación amplio, esto debido a que no se contaba con este proceso de prototipado tridimensional en los laboratorios de la Universidad.

El proceso de análisis para la matriz QFD se desarrolló como lo establece su guía (ver figura 5) y cuyos pasos se describirán a continuación.

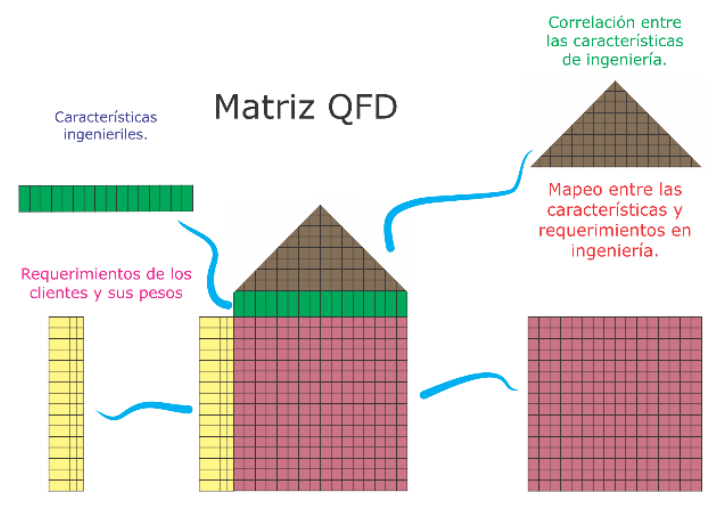

Fig. 5: Esquema de explicación de la Matriz QFD. Autor

\subsection{Requerimientos por el usuario final.}

En la Tabla 1, se puede observar, la implementación de la matriz QFD en el análisis de los requerimientos de los clientes, todos estos obtenidos a través de la encuesta implementada en Google Sheets (Cortés and Rodríguez 2014), en esta imagen también se puede observar la asignación de los pesos, y su importancia relativa, en donde parámetros como máquina precisa, exacta, estética, acabado, adaptable a materiales comerciales, fácil mantenimiento, y por último la interpretación de piezas en distintos formatos y su posterior fabricación tienen una aceptación mayor al 9\%, estableciendo estos elementos como fundamentales a la hora de construir la máquina, mientras que factores como el ser económica, portabilidad, área de trabajo amplia, por nombrar algunas, tienen una aceptación menor para el usuario final pero no indican que sean menos importantes, esto acotado a la idea principal de desarrollar una impresora 3D con un robot paralelo es bastante ideal, brindando así limitaciones en algunos aspectos y en otros facilidades de diseño y construcción.

Tabla 1. Requerimientos del cliente final, con su tabla de pesos.

\begin{tabular}{|l|l|l|}
\hline \multicolumn{2}{|c|}{$\begin{array}{c}\text { Importancia Relativa de Requerimientos en los } \\
\text { Clientes }\end{array}$} \\
\hline Requerimientos del Cliente & $\begin{array}{c}\text { Importancia } \\
\text { Relativa }\end{array}$ & $\begin{array}{c}\text { Ponderación } \\
\text { (\%) }\end{array}$ \\
\hline Máquina precisa y exacta & 8,28 & 10 \\
\hline Estetica y acabado & 7,5 & 9 \\
\hline Economía & 6,62 & 8 \\
\hline Rapidez de producción & 6,33 & 8 \\
\hline $\begin{array}{l}\text { Adaptable a Materiales } \\
\text { comerciales }\end{array}$ & 7,12 & 9 \\
\hline $\begin{array}{l}\text { Fabricación de cualquier } \\
\text { pieza }\end{array}$ & 7,31 & 9 \\
\hline Fácil manejo & 6,62 & 8 \\
\hline
\end{tabular}




\begin{tabular}{|l|l|l|}
\hline \multicolumn{3}{|c|}{$\begin{array}{c}\text { Importancia Relativa de Requerimientos en los } \\
\text { Clientes }\end{array}$} \\
\hline Requerimientos del Cliente & $\begin{array}{c}\text { Importancia } \\
\text { Relativa }\end{array}$ & $\begin{array}{c}\text { Ponderación } \\
\text { (\%) }\end{array}$ \\
\hline Área de trabajo & 6,81 & 8 \\
\hline Robustez & 6,73 & 8 \\
\hline Portabilidad & 5,4 & 6 \\
\hline Facil Mantenimiento & 7,11 & 9 \\
\hline $\begin{array}{l}\text { Interpretacion de piezas } \\
\text { en distintos formatos }\end{array}$ & 7,51 & 9 \\
\hline
\end{tabular}

\subsection{Análisis de los requerimientos funcionales.}

Para solventar los requerimientos del cliente, se plantean los requerimientos funcionales como soluciones técnicas inmediatas, los cuales se han expuesto en la tabla 2 con su respectivo orden de importancia relativa y ponderación porcentual.

Tabla 2: Requerimientos funcionales.

\begin{tabular}{|l|l|l|}
\hline \multicolumn{3}{|c|}{$\begin{array}{c}\text { Importancia Relativa de los Requerimientos } \\
\text { Funcionales }\end{array}$} \\
\hline $\begin{array}{c}\text { Requerimientos } \\
\text { Funcionales }\end{array}$ & $\begin{array}{c}\text { Importancia } \\
\text { Relativa }\end{array}$ & $\begin{array}{c}\text { Ponderación } \\
\text { (\%) }\end{array}$ \\
\hline Diseño Mecánico & 645,3 & 19 \\
\hline Software & 475,9 & 14 \\
\hline Materiales & 449,7 & 13 \\
\hline Manufactura Propia & 362,5 & 10 \\
\hline Control Eficiente & 309,4 & 9 \\
\hline Diseño Electrónico & 253 & 8 \\
\hline Dimensiones Optimas & 244,6 & 7 \\
\hline Actuadores & 208,7 & 6 \\
\hline Comunicación & 178 & 5 \\
\hline Interfaz HMI & 152,6 & 5 \\
\hline
\end{tabular}

Los requerimientos del cliente y las características de diseño se relacionan de manera fuerte (punto negro), moderada (punto blanco) o débil (triángulo), de acuerdo al análisis realizado con la matriz de calidad (ver figura 6). Esta relación deja ver el impacto de cada una de las características de diseño sobre las especificaciones del producto.

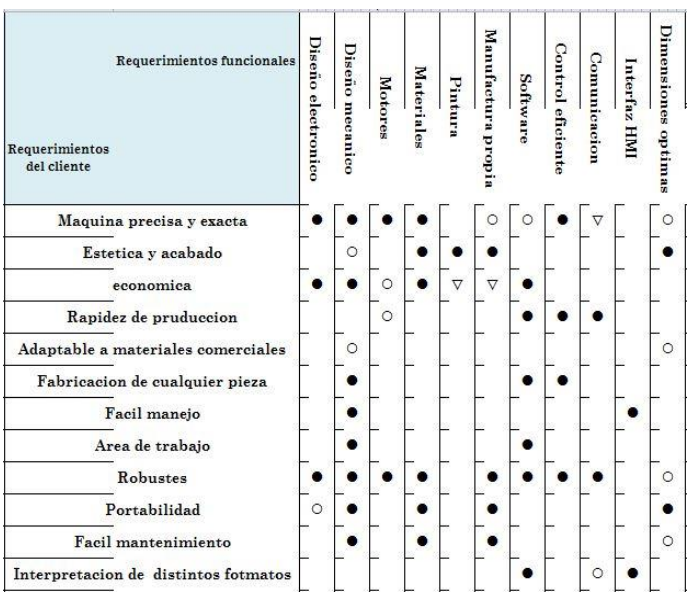

Fig. 6: Mapeo de relaciones de solución entre los requerimientos del cliente y los requerimientos funcionales.

Posteriormente se realiza una correlación entre los requerimientos expuestos para solucionar los problemas surgidos a partir de los expuestos por las peticiones de los clientes finales, por ejemplo los motores y el diseño mecánico se correlacionan positivamente debido a que se deben de tener buenos actuadores para que la máquina sea rígida y pueda desarrollar velocidades contrarrestando aspectos como las inercias producidas.

Los resultados analizados durante esta sección, arrojaron como necesidad el contar con una máquina de prototipado rápido con tecnología FDM (Modelado por Deposición Fundida) en la Universidad de Pamplona, a lo cual se le dio solución mediante el desarrollo de un robot paralelo para la impresión en 3D.

\section{DISEÑO ESTRUCTURAL}

Analizando los resultados obtenidos en el proceso de la sección de requerimientos (ver figura 7), se materializo la idea de desarrollar un robot paralelo para tareas de impresión en 3D, esta sección mostrara como se solucionaron cada uno de los requerimientos de los clientes.

Máquina precisa y exacta: la implementación de reducciones $(2,12: 1)$ acopladas entre el actuador y los eslabones iniciales del brazo, permiten suavidad en sus pasos, bajan la tasa de frecuencia de movimientos bruscos causados por altas inercias, además de esto, se utilizan motores PAP (Paso A Paso), los cuales tienen una resolución de 3200 pasos por revolución, lo cual brinda una capacidad alta de tener precisión y exactitud altas, para el eje 
$\mathrm{Z}$ se diseña un mecanismo husillo tuerca con la misma resolución de 3200 pasos por revolución en el motor, el tornillo que se diseña tiene un paso de $0.512 \mathrm{~mm}$ por vuelta.

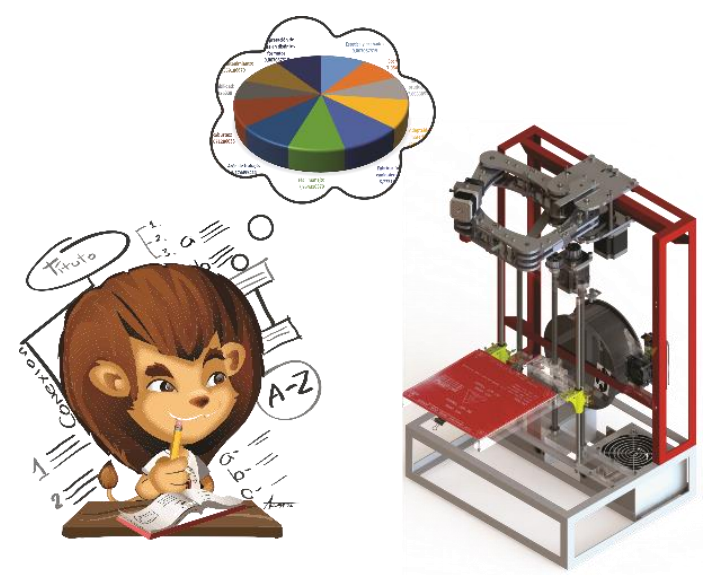

Fig. 7: Análisis y puesta en marcha del diseño partir de los resultados obtenidos a través de la matriz $Q F D$.

Estética y acabado: la presentación física de esta máquina se divide en varios factores:

1) El ser un tipo de robot diferente brinda un estigma a quien lo percibe.

2) Su estructura metálica en aluminio y acero brindan rigidez y empatía, por el hecho de percibir que es una máquina robusta $\mathrm{y}$ fuerte.

3) Por otra parte, las partes hechas en acrílico y ABS brindan estéticamente un contraste que llama la atención debido a sus colores y formas.

4) $\mathrm{Su}$ pintura roja $\mathrm{y}$ plateada hacen resaltar aún más las cualidades de la máquina.

5) Al ser una máquina descubierta completamente ofrece al usuario cierta comodidad para percibir lo que ocurre en el proceso, lo cual es agradable para estos.

Económica: esta máquina pretende ser un proyecto innovador, fiable y a bajo costo, entre otras características, lo económico de este proyecto radica en la implementación de partes recicladas de máquinas dadas de baja, lo cual hace que los costos de producción de este proyecto se vean disminuidos, y por ende se puedan utilizar para otro fin.

Rapidez de producción: es conocido que los robots tipo paralelos desarrollan altas velocidades, de acuerdo a los actuadores utilizados será su velocidad, sin embargo se espera que esta sea al menos un $50 \%$ más rápido que una de tipo cartesiano.

Adaptable a materiales comerciales: al ser presentado como un proyecto OpenSorce y OpenHardware se ha diseñado para que sus partes electrónicas sean de fácil acceso, y adaptables a estos proyectos, por lo cuales se ha comprado un extrusor capaz de extrudir materiales comerciales y convencionales (ABS, PLA) entre los más conocidos, los cuales tienen un rango de precios entre los $\$ 50.000$ y $\$ 100.000$ pesos colombianos por $\mathrm{Kg}$, que comparados con los $\$ 220.000$ de un $\mathrm{Kg}$ de material para impresoras profesionales, el ahorro es significativo.

Fabricación de cualquier pieza: Entre los requerimientos que se utilizaron para dar solución durante la encuesta, la selección de un software generador del código $\mathrm{G}$ de acceso libre hace que la máquina tenga mayor accesibilidad, factores, como la creación de material de aporte, reducir la velocidad en círculos pequeños, acabados y tamaño de las capaz permiten la fabricación de diferentes piezas complejas.

Fácil manejo: la máquina pretende ser accesible a los estudiantes de la Universidad de Pamplona, pero su manejo, pretende saber y/o adquirir habilidades de diseño y manejo de softwares tipo CAM para que pueda ser usada, por lo cual una solución además de la creación de una interfaz fácil de usar, será implementar cursos de manejo de la máquina para los clientes que lo soliciten.

Área de trabajo: es de conocimiento que el área de trabajo en robots paralelos es problemático dado a que suele ser reducido, debido al uso de cadenas cinemáticas cerradas, por lo cual se diseñan eslabones que puedan ser capaz de variar su longitud, en donde su distancia más corta es de 16 $\mathrm{cm}$, y la más larga de $20 \mathrm{~cm}$, lo cual permite adaptarlos a diferentes configuraciones y diferentes áreas de trabajo, en este caso, el área de trabajo es de $20 \mathrm{~cm}$ x $20 \mathrm{~cm}$, a lo cual la configuración de los eslabones en su extensión máxima se acopla perfectamente, además permite variar la posición de las singularidades.

Robustez: la robustez de la máquina en el diseño se solucionó mediante la implementación de materiales metálicos en los eslabones y la estructura, que brindan rigidez, mientras que en el eje $Z$ se utiliza una placa de acrílico de $11 \mathrm{~mm}$, lo cual ofrece firmeza para el soporte del mecanismo y de la piezas impresas. 
Portabilidad: el mecanismo al ser diseñado con materiales metálicos tiene un peso de $20 \mathrm{Kg}$, lo cual hace a la máquina difícil de ser transportada por una sola persona, siendo este un requerimiento poco apetecido por el cliente, no se tuvieron limitaciones a la hora de diseñar una máquina robusta su portabilidad es un poco más complicada.

Fácil mantenimiento: al diseñar un mecanismo en el que se pueda variar sus longitudes, este permite atornillar y desatornillar las uniones fácilmente, lo cual lo hace prescindible para un mantenimiento versátil sin necesidad de desarmar toda la máquina.

Interpretación de piezas en distintos formatos: al igual que la creación de piezas de cualquier geometría, se requiere que el diseño o pieza que se desea imprimir este en formatos .STL, .OBJ y/o .THING para que puedan ser utilizados en el software de CAM.

\section{RESULTADOS}

En esta sección, se encuentran los resultados obtenidos en cuanto a diseño y construcción de la plataforma.

\subsection{Diseño de la máquina}

El diseño de la máquina se basa en el modelamiento a través de la implementación de la metodología QFD, la cual permitió al grupo de trabajo, proyectar y contextualizar las ideas planteadas.

Se diseña una plataforma robótica paralelo de 3 GDL (Grados De Libertad), los 2 GDL para los ejes XY pertenecen al mecanismo 5R, mientras que el tercer grado de libertad envuelve el mecanismo husillo-tuerca, en el cual se contempla el uso de un extrusor tipo Bowden y una plataforma calefactora (HeatBed) para poder desarrollar tareas de impresión 3D, este diseño es visible en las figuras 8 y 9.

Cabe resaltar que el diseño del prototipo se desarrolla teniendo en cuenta los procesos de manufactura existentes en la Universidad de Pamplona (Fresadora CNC, torno, equipos de soldadura entre otros).



Fig. 8: Ensamblaje de la máquina, basado en los criterios de la matriz QFD.


Fig. 9. Partes de la máquina.

\subsection{Construcción de la máquina}

El desarrollo de la máquina, constituye la implementación de distintos procesos de manufacturas convencionales (torneadas, fresadas, soldadura) y no convencionales (impresión 3D), estos procesos, han sido enmarcados en los recursos establecidos en la planificación del proyecto, lo cual permite materializar la máquina teniendo en cuenta la ruta crítica establecida para él. 


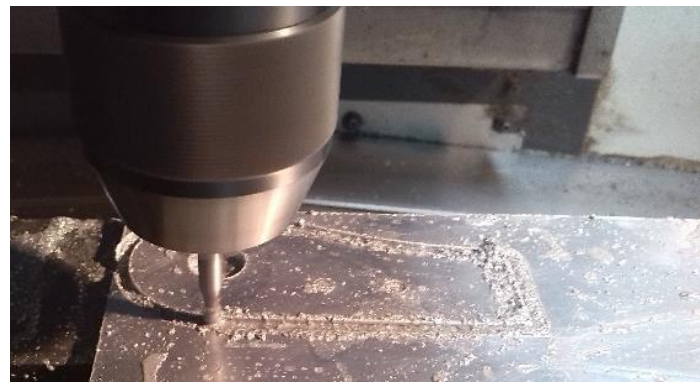

Fig. 10. Mecanizado de piezas en una fresadora CNC.

La fabricación de piezas en la centro de mecanizado LEADWELL V30, permitió el mecanizado para la mayoría de piezas de la máquina que tuviesen partes planas (ver figura 10), mientras que se usaron tornos para generar los cortes en cilindros, ejes y tornillos (ver figura 11).

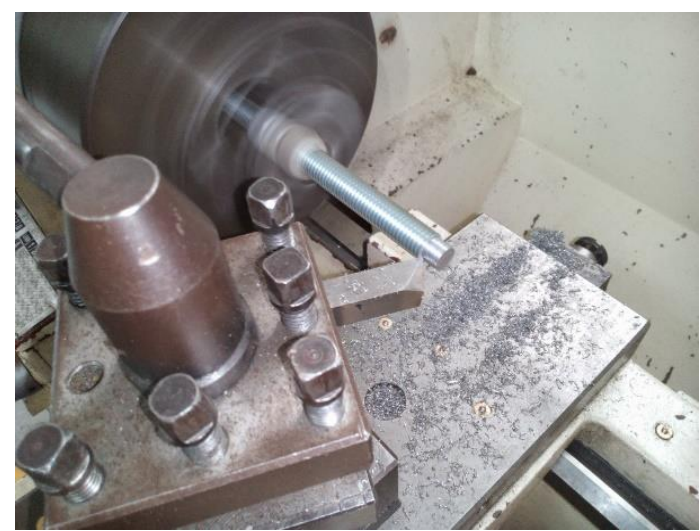

Fig. 11. Torneado de ejes para la máquina.

Por otra parte, en una impresora 3D se llevó a cabo la construcción de dos piezas vitales para el mecanismo del eje Z (ver figura 12), los cuales brindaron la posibilidad de mantener en su lugar los rodamientos lineales del sistema.

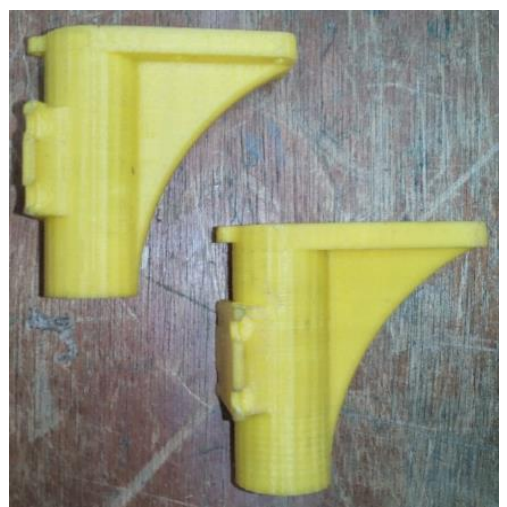

Fig. 12: Compartimiento para rodamientos lineales impresos en $3 D$.
El proceso de soldadura permitió elaborar la estructura de la máquina, al ser esta su columna vertebral, se posibilito el ensamble de las piezas y partes mecánicas de la máquina, este ensamble completo se puede observar en la figura 13.

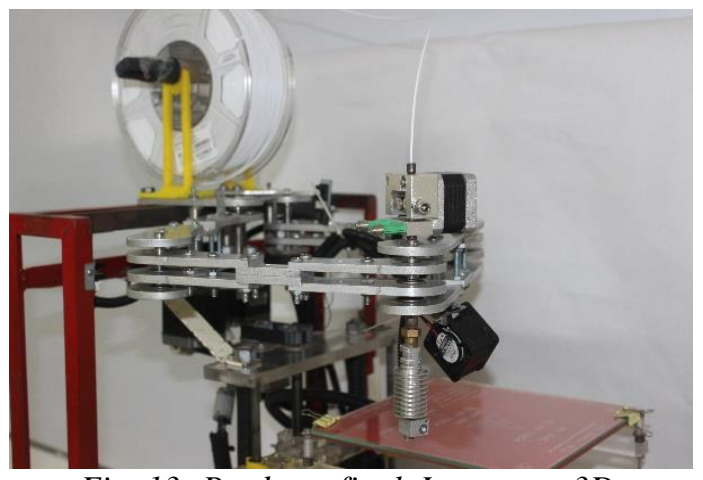

Fig. 13: Producto final. Impresora 3D.

\section{CONCLUSIONES}

Se ha sometido a la máquina a pruebas de funcionamiento, teniendo resultados alentadores de los cuales se puede concluir:

Esta máquina al ser un mecanismo de cadena cinemática cerrada brinda altas prestaciones como: alta repetitividad, precisión y velocidad.

La característica de variabilidad en las dimensiones del robot, permiten reajustar el espacio de trabajo y da lugar a un posible trabajo futuro de optimización dimensional.

Al involucrar la metodología de planificación QFD, se diseñó en función de los requerimientos del usuario, trazando las rutas de desarrollo que permitieron la ejecución del proyecto de manera satisfactoria.

\section{REFERENCIAS}

Chancusi, S., Navarrete, P. (2014), "Diseño Y Construcción de Una Impresora 3D AutoReplicable Controlada Inalámbricamente Para El Prototipado de Piezas Plásticas, Mediante Software Libre." Tesis doctoral.

Osgouie, K. G., "Meghdari, A., Sohrabpour, S. (2008). Optimal task-space manipulability of hybrid 4-DOF dual-arm CAM-lock manipulators". IEEE 5th International Symposium on Mechatronics and Its Applications, 2008. ISMA 2008. pp. 1-6. 
Bradley, D. A., \& Dorey, A. P. (1991). "Engineering design and mechatronics", IEEE Colloquium on In Mechatronics. Vol 2, pp. 2.

Burisch, Arne, S Soetebier, Jan Wrege. (2004). "Design of a Parallel Hybrid Micro-Scara Robot for High Precision Assembly." Mechatronics and Robotics, vol. 4, pp. 13701380.

Cervantes, J., Hernández, C., Rendón, J., (2000). "On the Workspace, Assembly Configurations and Singularity Curves of the RRRRR-Type Planar Manipulator." Mechanism and Machine Theory, vol. 35, no 8, pp 1117-1139.

Cortés, Eliseo, and Efraín Rodríguez. 2014. "Impresora 3D: Encuesta Requerimientos." Google Sheets. docs.google.com/forms/d/1od2P3JXbbdZPtJSw 70 wcegcbBNHbUkefa5UZ1OMFdY/viewform.

Cross, G A. (2014). "3D printing body parts : an overview of how additive manufacturing is shaping tissue engineering." Additive Manufacturing, pp 2012.

Dallalibera, F., y Hiroshi I. (2014). "Non-Singular Transitions between Assembly Modes of 2DOF Planar Parallel Manipulators with a Passive Leg." Mechanism and Machine Theory vol. 77, pp 182-197.

Hyun M. (2012). "Design and Control of HighSpeed Parallel Robot." 12th International Conference on control, Automation and Systems. pp 2139-2142.

Giberti, H., S. Cinquemani, and S. Ambrosetti. (2013). "5R 2dof Parallel Kinematic Manipulator - A Multidisciplinary Test Case in Mechatronics." Mechatronics vol. 23, no. 8 pp 949-959.

Goetsch, D., Stanley D., (1993) “Quality Function Deployment", Editorial Merrill, and Stephen Uselac.. $7^{\text {th }}$ Ed. Prentice Hall.

Huang, Y. Y., and K. H. Low. (2010). "Comprehensive Planning of Robotic Therapy and Assessment of Task-Oriented Functions via Improved QFD Applicable to Hand Rehabilitation." 2010 IEEE International Conference on Automation Science and Engineering, CASE 2010, pp 252-257.
Kahraman, C., Ertay, T., \& Büyüközkan, G. (2006). A fuzzy optimization model for QFD planning process using analytic network approach. European Journal of Operational Research, 171(2), 390-411.

Kim, W., Jun-Yong L., Byung J. (1997). “Analysis for a Planar 3 Degree-of-Freedom Parallel Mechanism with Actively Adjustable Stiffness Characteristics." Proceedings of International Conference on Robotics and Automation 3, pp 2663-2670.

Kostakis, V., Vasilis N., and Christos G. (2014). "Open Source 3D Printing as a Means of Learning: An Educational Experiment in Two High Schools in Greece." Telematics and Informatics. pp. 1-11.

Rodríguez Oscar Oswaldo, Pineda Pinto Ronald Fernando, Cárdenas Pedro Fabián. (2012). herramientas EJS 3D/MATLAB para el control del sistema no lineal aplicado al péndulo invertido sobre carro deslizante. Revista colombiana de tecnologías de Avanzada. 1 (19). Pág. $28-34$.

Liu, X., Jinsong W., Pritschow, G. (2006). "Kinematics, Singularity and Workspace of Planar 5R Symmetrical Parallel Mechanisms." Mechanism and Machine Theory, vol. 41, no. 2, pp 145-169.

Torres Clayton José, Archila John Faber, Tronco Mário Luiz, Becker Marcelo, Viera Porto Arthur José, Tiberti Alexander José. (2013). Estudio cinemático de una plataforma robótica para agricultura. Revista colombiana de tecnologías de Avanzada. 2 (22). Pág. 131 137

Pertuz, S.; Pena, C.; Riano, C., (2014) "RoboTender, A Robotic System Capable of Serving Drinks to Its User " III International Congress of Engineering Mechatronics and Automation (CIIMA), pp.1-5.

Caballero Amaury, Velasco Gabriel, Pardo García A. (2013). Differentiations of objects in diffuse databases. Revista colombiana de tecnologías de Avanzada. 2 (22), pp. 131 - 137. 